daily exercise being taken, the patient is, doubtless, placed in a very favourable position for gaining strength, and with it health. And in the remarks which I have made I by no means desire to make it appear that Davos is not a suitable winter station for certain classes of invalids ; but I wish particularly to show the necessity for the exercise of extreme caution in the selection of cases of lung affection which are allowed to proceed there. The fact that some persons suffering from phthisis go to Davos, gain in weight, and improve in their condition, affords no proof whatever that Davos is the best place to which such cases can be sent. A very large proportion of cases derive benefit, no matter to which of our health resorts they go. I have found the improvement wrought in the condition of many consumptive patients sent to Ventnor, Cannes, or San Remo, to be really marvellous. Turning to the medical reports of the Royal National Hospital for Consumption and Diseases of the Chest, located at Ventnor, I find that of the 474 patients suffering from chest affections treated in that institution during the years $1870-71-72-73,122$ were discharged from the hospital as improved, 161 much improved, 83 very much improved, 28 restored, 34 in much the same condition as when they entered, 22 were worse, and only 24 died. The gain in weight amounted to $2112 \mathrm{lb}$, and those who lost weight did so only to the extent of $286 \mathrm{lb}$. Of the 619 cases admitted during 1874 and 1875,130 left the hospital in improved health, 122 were much improved, 167 very much improved, 41 were restored, 91 remained in much the same condition, 37 became worse, and 31 only died, while the gain in weight amounted to $2117 \mathrm{lb}$. Of the 888 cases received into the hospital during the years 1876 and 1877,203 left it in improved health, 166 were much benefited, 236 very much so, 62 were restored, 77 remained in much the same condition, 94 became worse, and 50 died. No records were kept during these two years of the gains and losses in weight. Lastly, of the 501 patients admitted during the year 1878, 106 improved, 78 became much better, 150 very much so, 53 were restored, 36 remained in much the same condition as when they entered, 62 became worse, and only 16 died; 332 of these patients gained $1840 \mathrm{lb}$. in weight, and 124 patients, excluding those who died, lost $411 \mathrm{lb}$.

Now these results are very striking, and show the extraordinary benefit which most patients suffering from consumption and other diseases of the chest derive from change of climate, suitable diet, and appropriate medical treatment. They are, indeed, very favourable to Ventnor as a place of residence for persons suffering from affections of the lungs, but they by no means demonstrate that Ventnor is the best of all places to which such cases could be sent, neither does the fact that a certain proportion of the cases sent to Davos derived benefit therefrom, prove that it is the one place above all others for consumptives. Much more accurate and extended statistics than have hitherto been obtained, are required before any such conclusion is warranted.

Woburn-place.

\section{ON CERTAIN POINTS IN THE ETIOLOGY OF HIP-JOINT DISEASE.}

BY RICHARD BARWELL, F.R.C.S., SURGEON TO CHARING-CROSS HOSPITAL.

HIP-JOINT disease, surgically so-called, must be distinguished from certain diseases of the hip-joint, such, for instance, as arthritis deformans, or the form of suppuration which occasionally follows upon an exanthematous or enteric fever. The distinction is much more easily observed and pointed out at the bedside than made the subject of a definition. The disease with which we now have to do commences without prodroma, or with only slight loss of health in malaise, limping, and certain well-marked postures; it runs on with starting pains, a remarkable reversal of the characteristic position, wasting of the limb, abscess in various parts; and, after death or resection, such changes in the various constituents of the joint as we are in the habit of associating with the strumous diathesis are found. Although this disease frequently attacks those who are well fed and cared-for, yet it is more common among the poorer and less well-fed classes. Above all, this particular form of

malady is confined, or almost confined, to children under twelve years of age. It is, of course, well known that strumous joint disease generally is more common in earlier than in later life, yet at no other joint are such affections nearly limited to childhood. I look back on a long and wide experience, yet I do not know of a single case of hip disease proper commencing in adult life. Many relapses have I seen, even between the thirtieth and fortieth year of disease which had commenced in childhood, but not one originating after twenty-five, and only one after eighteen years of age. There must, then, be some peculiar cause or causes which render a malady almost unknown to adults so prevalent with children.

The anatomy of this articulation in early life is somewhat peculiar. The acetabulum is not, as in adults, hollowed out of a single bone, but is divided into three parts by as many lines of cartilage, which, radiating from near the centre of the cavity, like the limbs of a letter $Y$, give that name to the junction. Along both margins of each line enlargement by growth takes place; therefore, in six parts of the cavity within the synovial sac great plastic activity, together with hyperæmia, are continually going on.

The upper extremity of the femur of the newly-born babe is very different in form from the fully-developed bone the head is very large in proportion to the trochanters and shaft, the neck being quite undeveloped, so that the former seems sessile on the latter portion. Some time during the first year of life a bony nucleus appears in the upper part of the head, and during the fourth year an apophysal centre for the great trochanter. The small trochanter, whose ossification is much later, need not interest us here; but the peculiarities of relationship between the epiphysal head and the rest of the bone are very important. The subjoined cut, representing the section of a bone before union of its various parts has taken place, shows that the epiphysis does

FIG. 1.

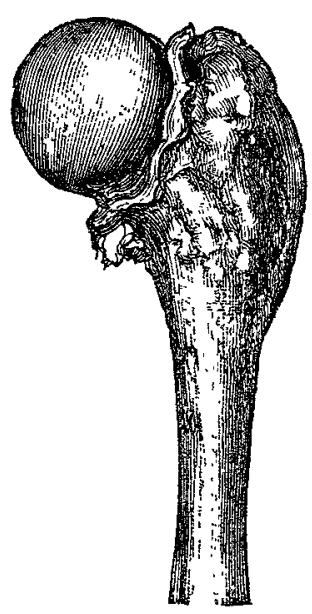

Upper end of femur; stillborn child. Head and upper end of shaft disproportionately large, indicating formative plasticity. Neck only marked by a narrow groove, giving attachment to synovial membrane and capsule.
FIG. 2,

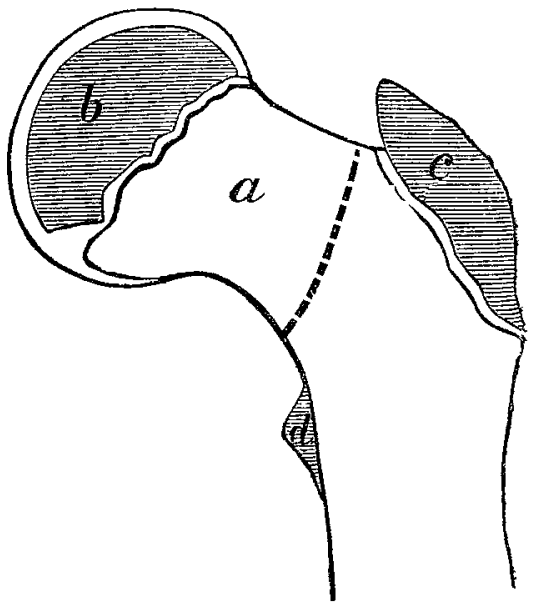

Section of upper end of femur ; boy aged sixteen. The diaphysis is left light; the additions are in tone. $a$, Shaft, neck, and diaphysal head. $b$, Epiphysal head. $c, d$, Large and small trochanters. The dotted line marks the synovial in. sertion.

not form by any means the whole of the head, but that all the lower and outer portion of this part and all the neck of the bone is derived from the diaphysal centre. Now, if we compare the upper femoral end of a newly-born child (Fig. 1) with that of a boy in his sixteenth year (Fig. 2), if we consider the place where the synovial membrane (represented by dotted lines) is attached, we see at once that the whole growth of the head takes place within the joint cavity; and not only so, but that all the neck-viz., more than an inch in length of a large bone-is formed entirely within the synovial membrane, which, as we see, encloses not merely the epiphysis, but also a large portion of the rapidly growing diaphysis. Let us contrast this condition with other large joints, as the knee. Here both the epiphysal junctions, save the anterior extremity of the femoral one, lie outside the joint; we see at once that hyperæmia, even disease, about these parts, may persist for a long time without affecting the joint itself. At the shoulder the inner portion of the epiphysal junction lies within the synovial sac, but, in the absence of a real neck to the humerus, how weak in comparison to that at the femur is the formative energy. At the hip, both pelvic and femoral growth lie entirely within the joint sac; that 
growth is most rapid, and any overaction, any inflammatory or other morbid condition, at this place is at once reflected as a joint affection. To my mind there is no doubt whatever that nearly all, if not all, the cases of infantile hip-joint disease originate in the bone, and more especially about the pelvic or epiphysal junctions; the proof of this position I must, however, for the present postpone.

But it may be said that all this proves only that, given a malady of the bones, hip disease must follow, yet shows no cause for the prevalence of osseous affections. Well, a certain causal connexion may be found in the fact that during the period when hip disease is frequent the child begins to throw its weight on the parts in question, learns to walk, and then becomes exposed to frequent falls and accidents. No doubt a certain number of cases may thus be accounted for ; but I confess to a strong opinion that some more potent agency has a larger share in the etiology.

A good many years ago I was struck with the fact that nearly all the boys we admitted for hip disease into Charingcross Hospital had congenital phimosis. In a short time this coincidence was found to be nearly, if not quite, constant. At last, in the middle of 1873 , I determined to note, in a hundred male cases of hip disease occurring in my private practice, or admitted into hospital, the presence or absence of this condition. For the sake of better classification phimosis was divided into three classes:-1 ist degree, the opening in the prepuce a mere pinhole, so that on retraction no part of the glans, or only a minute portion of the urethral lips, could be seen. 2nd degree, in which a considerable part of, or all but nothing beyond, the urethral orifice could be uncovered. 3rd degree, in which the prepuce, when retracted, uncovered some portion, but only a portion, of the glans. 4th degree, elongated prepuce projecting more than a quarter of an inch beyond the glans, but capable of entire retraction. 5th degree, normal.

Table of 100 cases of Hip Disease in Male Children under ten years of age.

1st degree. 2nd degree. 3rd degree. Elongation. Normal. 39

$17^{-\ldots} \quad 11$

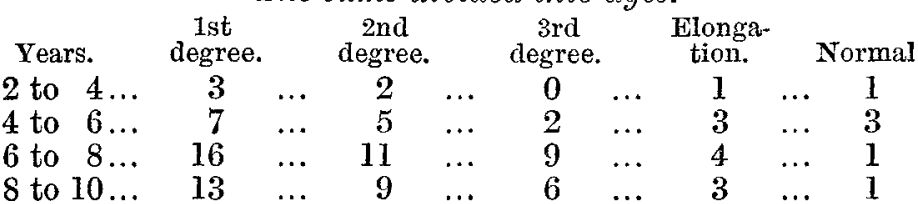

The first line of this table is very significant when it is considered that the cases are not picked or chosen, but represent every hip disease in the male that came under my notice, ${ }^{1}$ from the end of 1873 up to the middle of 1878 , when my number was complete. It will be observed that of these cases 83 have phimosis, that only 6 have normally formed prepuce, and that from complete, or the first degree of phimosis, to which class more than one-third of the cases belong, the number steadily declines to the normal. I would also point out that these are not fortuitous coincidences, because for two years at least before commencing tabulation this association was remarked. Furthermore, I asked my friend, Mr. Morrant Baker, to inquire for me about the prevalence of hip disease at the Evelina Hospital, which is largely used by Jews. He tells me that few children are there admitted for hip disease, and that most of those so received belong, not to the Jewish, but to the Christian community. I have appended also the second part of the table-that which divides the cases into ages-althongh I think in certain ways the division is not very reliable; for finding it impossible to ascertain from the accounts given by parents the exact period of commencement, the age at which I saw the child or admitted him into hospital has been recorded. Children of course came under my notice in all stages of the disease; hence the table shows nothing as to the time at which the malady commenced.

The important fact, however, is simply coincidence of phimosis and hip disease-a coincidence which I should never have dreamed of or imagined had it not been forced on my observation. Upon the mode in which the one influences the other I would rather not speculate further than to point out that phimosed children have facile, frequent, and often long-continued priapism; that this condition,

1 My colleagues at the hospital had kindly permitted me to make use also of their cases for statistical purposes. umnatural in the infant, must produce after a time a certain irritability or irritation of the lumbar spinal cord ; that from this part the various nerves of the pelvis and lower limb are given off; that the influence of spinal irritation on the trophic nerves is well known, and that just at this particular period large trophic changes are in progress about the hip-joint.

Of course, I have not overlooked the fact that hip disease also occurs in female children, though I believe less frequently than in the male. ${ }^{2}$ I regret exceedingly that I did not simultaneously tabulate such cases, as I am now engaged in doing; but this I can say with certainty, that in a large proportion of girls afflicted with hip disease will be found vulvitis, even vaginitis with or without discharge, and generally, I believe, produced in the first instance by threadworms creeping from the rectum to the vagina. In a certain proportion will be found protruding nympha or nymphæ covered by a cuticular surface. Further than this, as my numbers are incomplete, I am disinclined to go. The inference as to treatment of male cases in the earlier stages is obvious.

George-street, Hanover-square.

\section{A CASE OF}

\section{PAROXYSMAL HEMOGLOBINURIA, WITH} REMARKS ON ITS NATURE.

By STEPHEN MACKENZIE, M.D., F.R.C.P., ASSISTANT-PHYSICIAN TO, AND LECTURER ON PATHOLOGY AT, THE LONDON HOSPITAL.

(Concluded from $p .11 \%$.)

IT seems to me therefore, that the kidney is the seat of the blood destruction and blood discharge, and the next point to inquire into is the mechanism whereby these processes are brought into action. The phenomena of an attack of hæmoglobinuria are briefly as follow:-On exposure to cold, or in rare cases after depressing mental influences (in nearly all cases change from a warm to a colder temperature is the exciting cause of the attack), the patient turns pale, experiences a sense of chilliness, lassitude, and oppression, accompanied by a rigor of greater or less severity, or a goose-skin condition of the integument (cold stage). This is followed, after a longer or shorter space of time, by flushing of the surface, a sense of burning heat, ending in profuse perspiration (reaction). The first time the patient passes water after such a shivering it is observed to be black. He may continue to pass similar urine for some hours, but usually, or at least often, the water that he passes during or subsequent to the stage of reaction has the colour of health. Albumen is often present in the urine for some hours or even days subsequent to the appearance of blood. colouring matter.

It will be observed that in my patient exposure to cold, which excites the attacks of hæmoglobinuria, also induces attacks of urticaria. I think the urticaria furnishes us with an explanation, or at least strengthens that $I$ am about to express my belief in, of the mechanism of the hæmoglobinuria. In urticaria " first there is a morbidly sensitive skin; irritation is then applied, and is followed by spasm of the muscular fibres of the skin, and also of the capillaries, with subsequent dilatation of these vessels. Then follow hyperæmia or redness, escape of serosity, elevating the central part more than the outside of the lines, the whole constituting what is termed a 'wheal.' The tissues are passive." (Italics in original.) In cases of paroxysmal hremoglobinuria, during the cold stage of the rigor the cutaneous bloodvessels are in a state of spasm, and the blood, unable to reach the surface, is driven in upon the internal viscera. Whilst this blood fluxion is distributed more or less to all organs, the vascular arrangement of the kidney is such that its effects are peculiarly felt by the latter. The mechanism of renal secretion is so arranged that the renal capillaries are, even in health, subjected to a pressure greater than that of the systemic capillaries; and owing to this and

2 During the time that I was noting 100 male cases I saw only 73 female cases.

1 Skin Diseases, by Tilbury Fox, M.D., 3rd ed., p. 120. 